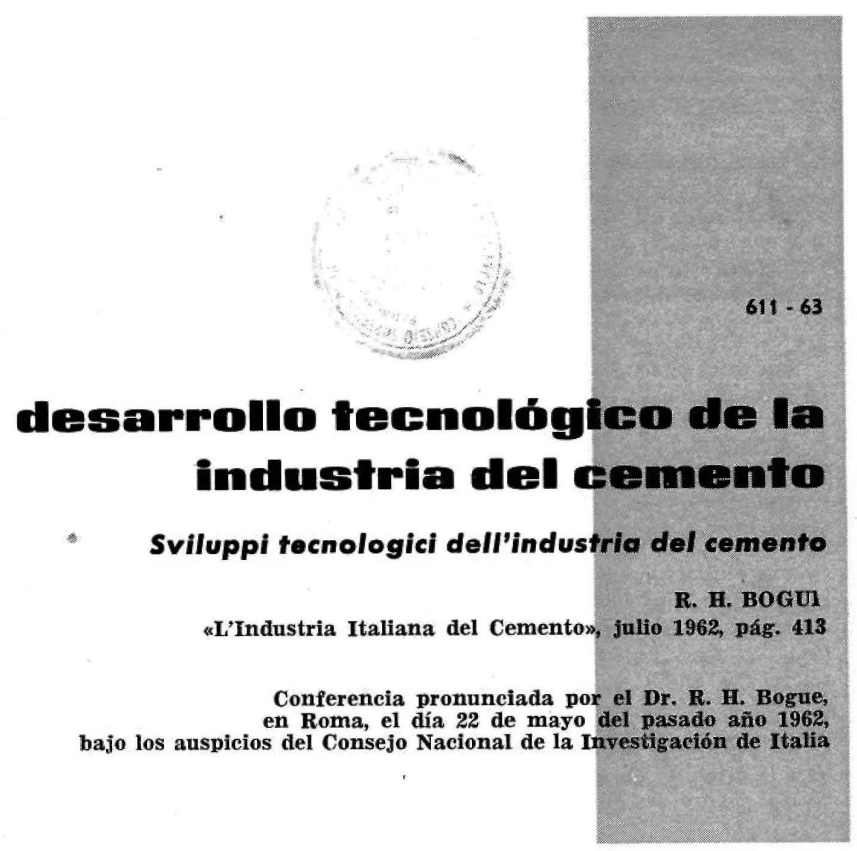

\title{
Panorama industrial
}

La producción mundial de cemento portland se ha más que duplicado en el transcurso del decenio comprendido entre 1950 y 1960 , habiendo pasado de 133 a 316 millones de toneladas métricas, lo que supone un aumento del $130 \%$. En los Estados Unidos el aumento no ha sido tan espectacular: la producción, en principio, fue de 39 millones de toneladas fabricadas en 150 fábricas, pasando a 57 millones producidas en 176 plantas, siendo el aumento porcentual del $46 \%$. El aumento en Italia, según la cifra del Bureau of Mines, es mucho más elevado. Efectivamente, la producción ha aumentado durante dich decenio de 5 a 16 millones de toneladas, es decir, al 220 por ciento.

Este notable aumento se ha debido más a los perfeccionamientos y ampliaciones de las fábricas ya existentes que a la construcción de nuevas plantas. En los Estados Unidos, el $46 \%$ de aumento en la producción se ha efectuado con tan sólo un $17 \%$ de aumento en el número de fábricas. La producción en las antiguas fábricas de cemento se ha incrementado mediante la construcción de hornos más grandes y la introducción de maquinaria más eficaz.

Ahora bien, los perfeccionamientos y mejoras en los medios para la producción del cemento son el resultado, y no la causa, de una demanda muy elevada. La construcción de carreteras, diques, edificios, puentes y viviendas ha hecho que el hormigón sea necesario en mayor cantidad. Hasta ahora el hormigón supera en la mitad a todos los demás materiales de construcción reunidos: hierro, acero, piedra, ladrillo, madera, arcilla de construcción, metales no férreos, vidrio, materiales plásticos. Ahora bien, un hormigón que cumplía su misión hace diez años ha sido superado por los progresos cada vez mayores de nuestra era. Una carretera adecuada para caballos y carros no lo es para automóviles, y una carretera considerada amplia para el modelo «T», no puede soportar el peso de los grandes vehículos de hoy día.

Me di cuenta de este estado de cosas recorriendo una antigua carretera llena de baches entre Nara y Osaka, en el Japón, hace ya unos años. Tenía que entrevistarme con un grupo de ingenieros en Osaka y, hasta el momento de dejar Nara ignoraba que se esperaba de mí una disertación. Por tanto, no había preparado ningún argumento, pero después de aquel memorable viaje tenía uno al alcance de mi mano.

Debo añadir que ahora la carretera está reconstruida. 
La autopista es lo que absorbe las mayores cantidades de cemento. En los Estados Unidos, el «Federal Highway Acb de 1956 ha comenzado un gran programa de construcciones de autopistas, el más importante programa de construcciones, que es dos veces más amplio que el mayor proyecto de obras públicas que se haya emprendido jamás en América. La cifra total a gastar en este proyecto se calcula en unos 104.000 millones de dólares, es decir, aproximadamente 8.000 millones de dólares anuales durante trece años.

En éste están comprendidas las redes federales, estatales y locales. El número de hombres empleados será de 442.000 . El cemento necesario será de cerca de 20 millones de toneladas métricas anuales y; además, se emplearán 4 millones de toneladas de acero, 10 millones de toneladas de productos bituminosos y grandes cantidades de otros materiales. Para darse una idea de la influencia del programa sobre la industria del cemento, basta decir que se utilizará más cemento para las carreteras en un año que se produjo en el año 1945 para todos los usos.

Sin embargo, esto no sólo proporcionará beneficios a los suministradores de materiales. E] impulso de tales gastos se convertirá en un mayor estímulo para la economía y las carreteras acelerarán la descentralización de la industria y el éxodo de la población en las zonas congestionadas. La carretera incita a la idea de expandirse siempre más y su desarrollo crea nuevos pueblos y ciudades.

Esta expansión industrial estimula el aumento de la producción de cemento. Los pequeños homos difícilmente podrán competir y tendrán que ceder el puesto a instalaciones más grandes. Es difícil que pase un año sín que oigamos hablar de un nuevo «horno más grande del mundo».

Adoptamos este superlativo para los grandes hornos de Holanda, Bélgica, Argentina, Portugal y Rusia.

Por no poder seguir el ritmo de la explosiva demanda de cemento, pequeñas cementerías y sociedades que no estaban en condiciones de producir en un mercado donde existe una gran competencia, han sido absorbidas por sociedades más importantes y han tenido que ser re. construidas desde la cantera hasta las instalaciones de expedición del cemento. Se har organizado muchas nuevas sociedades, y el capital y la competencia técnica europea han absorbido a la industria de todo el mundo. Han sido nuevamente elaborados los esquemas de los ciclos de fabricación, proyectada de ntuevo la maquinaria, y se han introducido modificaciones radicales.

Automatismo significa perfeccionamiento del control, e investigación y desarrollo significan estudio de ideas nuevas para la producción de cemento, que en un próximo futuro será capaz de revolucionar la industria.

En el transcurso de visitas precedentes a las fábricas de cemento europeas he observado notables diferencias con los sistemas americanos. Me he dado cuenta que muchas fábricas europeas se han desarrollado desde una honrosa antigutedad, mediante transformaciones sucesivas desde la producción de la cal hidráulica, y si bien se han madurado con el polvo de decenas y decenas de años, sin embargo, eran demasiado viejos cuando el cemento portland nacía. A veces la fábrica era de tipo artesano, siendo el propietario el que extrae la piedra del patio posterior e instala la maquinaria en la rosaleda. Pero todavía me sorprendió más el ver una fotografía en un periódico de Ceylán, en la que se veía una fábrica de cenento en China, que consistía en una fila de pequeños hornos verticales no más altos $v$ anchos que un hombre. La técnica americana no ha tenido que atravesar un período de desarrollo tan prolongado como en Europa. Por el contrario, se inició con todos los beneficios que suponía la experiencia del viejo mundo.

Otra notable diferencia entre la situación europea y la americana es consecuencia de que nuestra economía es totalmente diferente. En Europa la mano de obra es barata y el combustible es caro. En América sucede lo contrario. Por esta razón, nuestro rumbo y nuestro progreso se ha enfocado, bajo el punto de vista de reducir la mano de obra a costa de gastar más combustible, en tanto que las mejoras en Europa han tenido como fin primordial la eco- 
nomía de combustible. Por esto, aun cuando los hornos verticales han sido usados en Europa ampliamente y con éxito; la industria americana se ha basado exclusivamente en el horno rotatorio. Sin embargo, también en Europa la tendencia parece ser la vuelta al horno rotatorio. Así, en la fábrica de cemento de Lafarge de Teil he observado que una batería de 67 hornos verticales ha sido desechada, y en su puesto ha surgido, en la vieja planta, una moderna fábrica de cemento con homos rotatorios.

Siguiendo esta evolución de la industria del cemento dentro de la industria de la cal. la vía seca ha prevalecido hasta cerca de 1928.

Pero en aquella época los arquitectos e ingenieros prescribieron normas para una mayor uniformidad y alta calidad del hormigón, lo cual implica una mayor uniformidad y una más alta calidad del cemento. Tal producto era preciso hacerlo, sobre todo, con un crudo molido perfectamente homogeneizado, lo cual era difícil de conseguir por vía seca.

En vista de que se podía obtener una mezcla más uniforme mezclando con agua, se hace popular la vía húmeda, no obstante el gasto adicional de calor para la eliminación del agua añadida. Pero para evaporar el agua fue preciso cambiar el horno corto de la vía seca, por un horno más largo mediante la adición de una zona de secado los esfuerzos principales se dirigieron hacia la obtención de un intercambio de calor $\mathrm{y}$, con tal fin, se idearon numerosos dispositivos.

Hacia el año 1945 se introdujeron, en los sistemas de alimentación de los hornos por vía seca, métodos de fluidificación y homogeneización mediante los cuales la materia prima se mezclaba íntimamente como la pasta del proceso húmedo. El resultado de esto fue una inversión de las tendencias y que la mayor parte de las nuevas fábricas de cemento adoptasen la vía seca.

\section{La maferia prima}

\section{Dosificación}

Se ha escrito mucho sobre la dosificación de la materia prima para producir un cemento de la calidad y composición deseada. Desde los albores de esta industria, sabemos que el alejarse de una fórmula de dosificación convencional tiene como resultado un cambio desastroso en las propiedades del cemento.

Las causas han sido descubiertas muy «a posteriori» mediante investigaciones sobre el equilibrio de fases, a consecuencia de lo cual se ha podido apreciar que una disminución de un $1 \%$ en el $\mathrm{CO}_{8} \mathrm{Ca}$ de la mezcla tiene como consecuencia reducir en un $14 \%$ el contenido de $\mathrm{C}_{3} \mathrm{~S}$. Igualmente, la introducción de un $1 \%$ de cenizas de carbón puede rebajar el contenido de $\mathrm{C}_{3} \mathrm{~S}$ en más de un 6 por ciento.

Esto era un viejo problema que ya se encuentra resuelto. Creemos oportuno indicar en este párrafo, una vez más, que es necesaria una gran exactitud en el control para evitar que los valores que obtengamos se determinen más en el polvo del aire del laboratorio que en la cal de la pasta en cuestión.

\section{Fosfotos}

Los tipos de materias primas adecuados para la producción de cemento son demasiado conocidos para requerir su enumeración.

Pero a veces se presentan componentes extraordinarios que requieren especiales consideraciones, como, por ejemplo, la piedra fosfática en Uganda. 
La presencia de fosfatos en la materia prima del cemento se ha reconocido tolerable en pequeñas proporciones, pero inadmisible en grandes cantidades. En tal caso los fosfatos solubles en el cemento retardan fuertemente el fraguado y reducen las resistencias iniciales. Los fabricantes han encontrado dificultades para cocer u cínker con bajo cortenido de cal libre.

La química de los sistemas de fosfatos aplicables a los cementos ha sido estudiada por algunos investigadores, obteniéndose como resultado que, hoy día, se pueden controlar mejor las operaciones cuando están presentes los fosfatos. La composición de la solución sólida de los fosfatos es, aproximadamente (no exacta), $\mathrm{C}_{8} \mathrm{P} \cdot \mathrm{gC} \mathrm{S} \cdot 3 \mathrm{C}$. Un hecho importante para los fabricantes es que se ha encontrado que por cada un $1 \%$ de $\mathrm{P}_{2} \mathrm{O}_{\mathrm{s}}$ presente, el $\mathrm{C}_{3} \mathrm{~S}$ se reduce en cerca de un $10 \%$. La cantidad de $\mathrm{CaO}$ correspondiente según el cálculo de saturación en cal, viene reducido en poco más del $1 \%$ para cualquier porcentaje de $\mathrm{P}_{2} \mathrm{O}_{5}$ presente. Esto signifiça que los cálculos, bien sean por la cantidad de $\mathrm{C}_{3} \mathrm{~S}$ o bien por la saturación en cal, deben ser modificados en presencia del $\mathrm{P}_{2} \mathrm{O}_{5}$; de lo contrario, queda en el clínker exceso de cal libre o un contenido de $\mathrm{C}_{3} \mathrm{~S}$ insuficiente. La reduccićn en $\mathrm{C}_{3} \mathrm{~S}$ y el aumento de cal libre explica el descenso de resistencias iniciales, notablemente reducidas, y la difícil cocción de Jos cementos que contienen fosfatos.

Utilizando las ecuaciones dadas por Nurse y generalizadas por Steinour podemos tener ahora clínker con un contenido en $\mathrm{P}_{2} \mathrm{O}_{3}$ del 2 al 2,5\%, bajo contenido de cal libre y buen porcentaje de $\mathrm{C}_{5} \mathrm{~S}$.

Sin embargo, hay divergencia de opiniones sobre si un cemento hecho con tal clínker servirá en la práctica. Cualquier fábrica de cemento debe hacer pruebas muy detalladas antes de emprender la fabricación de cemento con tal porcentaje de $\mathrm{P}_{2} \mathrm{O}_{5}$.

\section{Molienda}

Tanto la materia prima como el clínker'se molían en principio en circuito abierto. Una prueba efectuada en nuestro laboratorio hace ya muchos años demostró que sólo se observaban ligeras reducciones en la aptitud a la cocción como consecuencia del aumento en las dimensiones de la caliza de 200 a 100 mallas ( 74 a 149 micras), pero sí se observaban grandes reducciones aumentando las dimensiones del polvo de cuarzo dentro de este campo. Esto significa que el cuarzo o la sílice en la materia prima debe molerse de manera que, prácticamente, pase un $100 \%$ por el tamiz número 200. Mezclas con materiales esquistosos o con arcilla cuecen más fácilmente que con el cuarzo, pero lo que clinkeriza mejor que todo lo demás es una marga donde los componentes se encuentran en contacto muy íntimo.

Un interesante ejemplo de este efecto se me presentó hace unos años en el curso de una visita que hice a una fábrica de cemento próxima a Londres. Los técnicos estaban muy preocupados por las bajas resistencias iniciales, y porque la cal viva persistía en su clínker aun cuando el crudo tenía un contenido en cal muy por debajo de la saturación en cal calculada. Examinando el clínker petrográficamente en placas muy delgadas, se observaron numerosas aureolas de $\mathrm{C}_{2} \mathrm{~S}$ próximo a zonas vacías, y a cierta distancia muchos granos de cal libre. Los huecos fueron en cierto momento gránulos de sílice, pero estos granos se combinaron con el óxido cálcico circundante para formar silicato bicálcico. El óxido cálcico necesario para combinarse con el $\mathrm{C}_{2} \mathrm{~S}$, por encontrarse en estado sólido y a una distancia muy grande del $\mathrm{C}_{3} \mathrm{~S}$ a fin de que pudiese tener lugar la difusión rápidamente, no estaba en condiciones de reaccionar para formar el $\mathrm{C}_{3} \mathrm{~S} y$, por tanto, resultaba imposible la combinación, $y$, por ser pobre en $\mathrm{C}_{3} \mathrm{~S}$, el cemento tenía más bajas resistencias iniciales. Las soluciones a adoptar eran las siguientes: o eliminar los granos gruesos de sílice, con modificación de la mezcla, o separarlos, molerlos nuevamente y mezclarlos otra vez con la alimentación del horno. Actualmente, esto se ha eliminado $y$ el inconveniente ha desaparecido.

Nuestros estudios se han dirigido hacia la necesidad de la molienda de materia prima en circuito cerrado, que actualmente es una práctica generalmente adoptada. Respecto a la molienda del clínker existen divergencias de opiniones. Las pruebas realizadas en los laboratorios 
de la Portland Cement Association han indicado que la molienda en circuito cerrado da como resultado un aumento en la economía del proceso, especialmente para las finuras más altas. Se observó una pequeña diferencia en las resistencias y un ligero empeoramiento en la durabilidad, medida mediante la resistencia de probetas al hielo y deshielo. El empeoramiento fue considerado insignificante al compararto con las notables mejoras obtenidas con el arrastre por aire.

Pienso, sin embargo, que sean importantes algunas otras consideraciones. El cemento portland es el único, entre los materiales de construeción, que posee intrínsecamente márgenes de seguridad que tienden a mejorar el hormigón a medida que transcurre el tiempo y a restaurar parcialmente su estructura envejecida. Uno de estos factores de seguridad es su contenido en $\mathrm{C}_{2} \mathrm{~S}$, un componente que continúa ganando resistencias durante muchos años si existe agua disfonible. El otro factor de seguridad es la presencia de partículas de diversas dimensiones (si se efectúa la molienda en circuito abierto), lo cual hace que la hidratación continúe más adelante durante un determinado período. Las partículas más pequeñas se hidratan y endurecen rápidamente, pero las de mayor tamaño pueden requerir algunos años para completar su hidratación. Por este motivo un cemento producido en circuito abierto, que contiene una amplia granulometría, posee un margen de seguridad a largo plazo que no lo tiene el cemento molido en circuito cerrado.

\section{Fluidificanfes de la mezcla}

El agua añadida durante la molienda del crudo en instalaciones por vía húmeda tiene que ser eliminada a razón de 650 a $1.400 \mathrm{~kg}$ de agua por tonelada de clínker. Unas mezclas requieren más agua que otras; pero el proceso será más económico, según el grado en que puede reducirse el agua antes de llegar al homo. Se ha experimentado un gran número de sustancias químicas defloculantes (fluidificantes de la pasta) en las fábricas y en el laboratorio.

Estas sustancias permiten que la pasta pueda ser bombeada normalmente con una cantidad de agua por debajo de la normal. De unos 300 productos químicos examinados, sólo 30 ó 40 de ellos han sido útiles con uno o varios tipos de pastas.

En Alemania se han empleado carbonatos e hidróxidos alcalinọs, silicato sódico, jabones, aceites y ácidos húmicos y lignínicos.

En Suecia se ha patentado un producto a base de turba tratada con sosa cáustica; en Estados Unidos: sales metálicas del ácido lignin-sulfónico, ácidos grasos sulfonados, compuestos de creosota sulfonados y hexametafosfato alcalino con carbonatos alcalinos. Un grupo de 273 sustancias tensioactivas se examinaron en la Portland Cement Association, comprendiendo materiales no iónicos, aniónicos, catiónicos, inorgánicos e inclasificables. Puesto que las pastas crudas son de origen diverso y pueden presentar diferentes reacciones a los agentes químicos, se utilizaron seis pastas de diversos tipos. Los resultados demostraron que, en general, las sustancias más útiles y econónicas son las soluciones sulfíticas resultantes de la fabricación de la celulosa, el carbonato sódico, el silicato sódico y ciertos fosfatos. Se han encontrado que, de estos productos, el tripolifosfato sódico y el pirofosfato tetrasódico en un 0,05 a un $0,10 \%$ ha permitido, en muchos casos, transportar la pasta con contenidos de humedad considerablemente bajos sin disminución en la facilidad de bombeo ni otra dificultad en la marcha de la instalación. $\mathrm{El}$ aditivo puede ser añadido a la arcilla en el desleidor o alimentado en seco, al molino con tornillo o cinta.

La acción de sustancias dispersantes, sin embargo, no es beneficiosa en todas las instalacio. nes y algunas veces surgen dificultades, tales como atascos en los molinos y en las tuberías. disminución de la facilidad de bombeo, formación de anillos de fango, o aumento en las pér. didas de polvo. En cada fábrica se deben efectuar experiencias con los propios materiales y después hacer el balance de las ventajas debidas a una disminución en el costo de la molienda. al menor consumo de combustible y al aumento de producción del horno en comparación con el aumento de dificultades en la marcha de la fábricá. 
La extracción de agua mediante filtrado por vacio en grandes tambores y la introducción de la torta que sale del filtro en el horno, es un procedimiento bien conocido y no merece ser estudiado aquí.

\section{La cocción}

\section{Hornos y cambledores de calor}

Se han efectuado muchas variaciones en la conducción del horno. No hace mucho tiempo era costumbre mantener constante el gasto de combustible, controlando la cocción mediante variaciones en la válvula de tiro y en la velocidad de giro del horno. Más recientemente, se ha actuado manteniendo constante la velocidad del horno y la alimentación, controlando la cocción por variación en la cantidad de combustible.

Creo necesario llamar repetidas veces la atención sobre la importancia de conducir el horno de una manera uniforme y estable para la economía y calidad del clínker. La composición, finura y contenido de agua de la mezcla cruda debe ser mantenida constante. No se debe alterar el tiro, puesto que esto determina la posición de la zona de cocción y tales cambios favorecen la formación de anillos de clínker y la producción de un material con contenido variable de cal libre.

La formación de ios anillos de clínker puede atribuirse a muchas causas, pero la falta de uniformidad en la composición y en la conducción del horno son, sin duda alguna, las más importantes. Si se sobrepasa la producción unitaria normal, el hornero aumenta la cantidad de combustible y la cantidad normal de aire introducida no suele ser la suficiente para obtener una combustión completa. Esto da como resultado un clínker poco cocido. Las alteraciones en la composición, o en la finura, o en el contenido de agua de la mezcla cruda, variaciones en el grado de saturación en cal, o de velocidad del horno, o en la cantidad de aire introducido, o en la longitud de la llama, o en las características del combustible, o en la introducción de polvo recuperado, todos tienen su efecto. Ahora bien, en cualquier caso una desviación brusca de la uniformidad, de las condiciones ideales de marcha, es responsable de la formación de anillos de clínker.

El haberse reconocido la necesidad de una conducción uniforme es la causa de la introducción de numerosos instrumentos para vigilar el horno, tales como analizadores de gases de salida, pirómetros registradores para indicar continuamente la temperatura en la zona de cocción y en el extremo de alimentación, registradores de la velocidad del horno, reguladores de la relación aire-combustible, contadores registradores del gas.

El homo Lepol, que ha tenido un gran éxito en Europa por su bajo consumo de combustible, no es popular en América puesto que ya se ha probado la versión ACL de doble paso. La razón parece que debe atribuirse a una mayor cantidad de mano de obra precisa para su conducción. Una innovación en la marcha del horno Lepol fue introducida por la Compañía de Cementos de Loma Negra, en Argentina, que funcionaba por vía húmeda. Se filtraba la pasta para producir una torta que después se granula e introduce en la parrilla de precalentamiento del horno.

Los resultados han sido satisfactorios y ha permitido a una fábrica que funcionaba por vía húmeda trabajar con un consumo de combustible comparable al de una vía seca.

He visto el primer precalentador Humboldt, por suspensión, funcionando, en Bremen, en el año 1952, y lo describí en mi discurso de apertura en la P.C.A. La Sociedad Fuller ha obtenido recientemente la licencia de fabricación y lo ha montado en un considerable número de fábricas de cemento. La razón de la popularidad de este dispositivo es el de una gran economía de combustible, un notable aumento de la producción, un mínimo entretenimiento de mano de obra para el funcionamiento y la posibilidad de economizar espacio, utilizando un horno corto de vía seca. Tales precalentadores pueden hacer que sigan usando los hornos cortos para vía seca que actualmente no están en condiciones de hacer frente a la competencia, a causa de su 
baja productividad y deficiente eficacia. Este conjunto resulta más eficiente que los hornos modernos de gran longitud por vía seca que presentan altas temperaturas en los gases de salida. Se estima que un horno de cerca de $3,5 \mathrm{~m}$ de diámetro y $45,5 \mathrm{~m}$ de longitud puede producir más de $500 \mathrm{t}$ de clínker diarias.

La práctica ha demostrado que el contenido de álcalis en el clínker cuando se introducen nuevamente en el horno los polvos recuperados, es sólo ligeramente más alto que el de un clínker producido en un horno tradicional en el que no se recuperan los polvos. Y cuando es necesario enviar a la chimenea los gases limpios, el precalentador de cuatro etapas tiene la ventaja de reducir las dimensiones de la instalación para la captación de polvos, ya que el volumen final de los gases es reducido y su temperatura está muy por debajo de la temperatura de seguridad para el funcionamiento.

Otros tipos de cambiadores de calor parecidos han sido diseñados con el fin de economizar calor. El procedimiento Miag permite la salida de los gases y polvos del horno a una temperatura de cerca de $750^{\circ} \mathrm{C}$. haciéndoles pasar a través de un tambor que gira lentamente y que contiene cilindros de unos $10 \times 10 \mathrm{~cm}$. El contenido de agua de la pasta se reduce en el tambor hasta cerca de un $7 \%$. La Sociedad $F$. L. Smidth ha introducido un procedimiento similar, pero que se diferencia de éste en que el tambor con los cilindros metálicos está incorporado formando parte integrante del horno. La Sociedad Vickers coloca en la cabeza de alimentación del horno un secador, que consiste en un doble caracol sujeto a la chapa y que sostiene las cadenas. El agua de la pasta se reduce, por lo menos, un 1,8\%. El secador Holderbank se ha proyectado para aumentar la transmisión de calor por convección entre el clínker y los gases de combustión, así como entre el crudo y los gases del horno. Un sistema de paletas de elevación voltea la carga y la deja caer atravesando la corriente de gases. Mediante una fila de deflectores, se produce un encauzamiento de los gases que aumenta la velocidad de flujo de éstos. Se afirma que se observa una economía en el combustible de más de $20 \%$, pero el procedimiento requiere utilizar nódulos sólídos y muy duros en la materia cruda.

Desde hace mucho tiempo se ha teconocido que el horno vertical es muy adecuado para pequeñas producciones y es muy económico en consumo de combustible. Las objeciones que se hacen respecto a su utilizacién son que el clínker que se obtiene con él no es homogéneo y el cemento poco uniforme. Recientes mejoras en las dimensiones y en la forma del horno vertical pueden rechazar estas objeciones. Mediante una sección superior de forma cónica para absorber la contracción de la carga durante la cocción y una parrilla escalonada en el fondo para que la descarga sea uniforme, Spohn afirma que el horno vertical puede actualmente producir un clínker que responde con pleno éxito a las normas de calidad más severas.

Berz, en Karlstadt, ha proyectado un precalentador consistente en un horno vertical en el cual los nódulos se desecan, y calcinan parcialmente, antes de pasar a un horno rotativo corto para la sinterización. Creo que actualmente se encuentra en periodo experimental.

La «Lurgi Company», de Dotterhansen, ha adoptado, para ser utilizada en la fabricación de cemento, una parrilla sinterizadora que ha sido utilizada en la tostación de minerales metálicos.

La instalación es única, pues en lo que respecta a materia prima se trata de un esquisto petrolífero, parte del cual se destila para recuperar el petróleo, que, a su vez, se usa en la cámara de combustión de la parrilla sinterizadora. El crudo contiene minerales esquistosos crudos y también cok triturado. La mezcla se distribuye sobre una parrilla móvil, de unos $2 \mathrm{~m}$ de ancho, que recorre lentamente una distancia de cerca de $24,5 \mathrm{~m}$, primero bajo unas cámaras de combustión y después atraviesa una cámara donde los gases calientes y el aire son aspirados por debajo de la parrilla. Tanto la producción como la economía de combustible son satisfactorias.

\section{Revestimiento de los hornos}

El revestimiento cumple dos importantísimas funciones: Primero, aísla la chapa de los gases de la combustión, que, de otro modo, en la zona de cocción, podrían deformar o fundir el acero; esta condición requiere un refractario de elevada resistencia al calor, baja conductivi- 
dad térmica y que contenga sólo vestigios de componentes fusibles a la temperatura que ha de trabajar. En segundo lugar, el revestimiento sirve como medio de transferencia del calor entre el gas caliente y los componentes del crudo; esto implica que el refractario puede almacenar calor en contacto con los gases de combustión durante una parte del giro del horno y lo ceda al crudo cuando entra en contacto con él, en una segunda parte del ciclo de rotación.

Una conducción del horno satisfactoria requiere una cierta compensación entre las dos direcciones opuestas del fijjo térmico que se manifiestan en cada giro del horno.

Se han utilizado muchos tipos y composiciones del revestimiento para la zona de cocción en el curso de la fabricación del cemento, pero en los últimos años se ha orientado hacia dos tipos preponderantes: refractarios de alto contenido en alúmina y refractarios magnesianos. Debido a su composición, el primer tipo viene definido, ordinariamente, como «refractario ácido» y el segundo tipo como «refractario básico». Los refractarios aluminosos son los que tienen más antiguiedad en su utilización y, además; son los más económicos. Son los menos refractarios y contienen componentes fusibles que tienden a fundir los ladrillos aislados convirtiendo el conjunto en un bloque monolítico. Una costra de los componentes del cemento, esencial para la buena marcha del horno, se forma fácilmente sobre el revestimiento refractario nuevo; pero, con todo, los refractarios aluminosos no son tan resistentes como los magnesianos y la consecuencia normal es que el revestimiento del horno tenga una vida más corta.

Los refractarios magnesianos han sido mejor acogidos por poseer un poder refractario más elevado y porque normalmente prestan servicio durante una campaña más larga. Sin embargo, tienen el inconveniente de soldarse con mayor dificultad y hacer más difícil la formación de costra. Para neutralizar estos inconvenientes, en la práctica, se ha recurrido al empleo de chapas metálicas, colocadas entre las caras de dos ladrillos. Durante el funcionamiento, estas láminas se funden, en parte, y también se oxidan colaborando a la aglomeración de los ladrillos, a la formación de costra y a la consolidación del revestimiento. Pruebas recientes indican cierta ventaja en la duración del revestimiento y en el consumo de combustible si se utilizan sistemas de láminas distanciadas una pulgada $(25,4 \mathrm{~mm})$ de la chapa del homo. (Patente Americana U.S.A. número 2.895.725 F. J. Anderson 21 julio 1959). Con este procedimiento se pierde menos calor por conductividad y radiación y se mantiene más calor para obtener las reacciones de formación del clínker.

Una idea clara sobre la importancia del proceso de cocción se puede tener si se estudia la termodinámica de la formación del clínker.

- Si se tiene en cuenta el principio de que el flujo térmico va en contracorriente respecto al crudo, los gases calientes de la combustión en el extremo superior del horno evaporan rápidamente el agua de la pasta inmediatamente de entrar ésta en el horno. Esto se consigue con un sistema de intercambio térmico, que corrientemente consiste en unas cadenas dispuestas en la cabeza de alimentación del horno. A medida que el crudo desecado desciende, como consecuencia del giro del horno, va encontrándose con intensidades de calor mayores. El anhídrido carbónico se libera a una temperatura de unos $900^{\circ} \mathrm{C}$, y cerca de los $950^{\circ} \mathrm{C}$ se manifiestan cambios estructurales en el óxido de calcio que aumentan su reactividad.

La aparición de fase líquida en el material se manifiesta de improviso a unos $1.280^{\circ} \mathrm{C}$, que es la temperatura de la mezcla eutéctica de punto de fusión más bajo. Con el aumento de calor, la temperatura permanece constante hasta que se forma todo el líquido de composición eutéctica que se puede crear en relación con la composición de la mezcla.

Después de esto, y debido a la incorporación de más calor, aumentará la temperatura hasta que se llegue a la de otra mezcla eutéctica, estabilizándose otra vez la temperatura mientras se forma el líquido correspondiente a la composición del segundo eutéctico. Estas alternativas se repiten hasta que se alcanzan las más altas temperaturas. La fase líquida, finaimente, contiene toda la alúmina, el óxido férrico de la mezcla y pequeñas cantidades de sílice y cal. Estas cantidades varían según la composición y la temperatura, pero a $1.480^{\circ} \mathrm{C}$ puede ser del 20 al $30 \%$ del material sinterizado. 
Las reacciones entre los componentes de la mezcla tienen lugar, principalmente, en la fase líquida. Algunas reacciones se producen a temperaturas relativamente bajas, del orden de unos $1.300^{\circ} \mathrm{C}$; pero si el tratamiento se diera por terminado en ese momento, el producto se habría combinado sólo parcialmente, existiendo mucha cal libre y presentando muy poca hidraulicidad. $\mathrm{Si}$ se habían formado los aluminatos y los compuestos de hierro estará presente el silicato cálcico, pero existirá una exigua cantidad del principal compuesto del cemento: el silicato tricálcico.

La reacción final-la más importante-es la combinación de la cal con el silicato bicálcico para formar el silicato tricálcico. La reacción se realiza muy lentamente hasta que el «sinter» alcanza de 1.380 a $1.480^{\circ} \mathrm{C}$. A esta temperatura se inicia una reacción exotérmica, que se traduce en una repentina incandescencia cuando los componentes reaccionan entre ellos hasta aumentar su temperatura interna de 100 a 200 grados centígrados.

A medida que la masa sinterizada avanza más hacia la zona caliente su temperatura aumenta, bien por la acción directa del gas de combustión o bien por la conducción e irradiación del revestimiento o costra. El calor transmitido por el revestimiento o por la costra es la fuente más importante de calor transferido al material en curso de sinterización. Dado que el clínker se mueve siempre hacia abajo mientras que el revestimiento sólo gira, el revestimiento con su costra absorbe el calor durante la fase de alejamiento del sinterizado, pero, a su vez, caldea al material al entrar en contacto con él. Por tanto, excepto cuando se produce la reacción exotérmica, la superficie del revestimiento o de la costra estará más caliente que el sinterizado durante la mayor parte de la rotación. En este punto, el sinterizado puede estar más frío que el revestimiento unos 100 grados centígrados.

Si una parte del sinterizado llega a alcanzar el punto exotérmico de ignición, la reacción, a no ser que el calor absorbido de la llama o transmitido por el revestimiento sea de tal interjsidad que mantenga la temperatura de incandescencia, se extinguirá. Si el calor del revestimiento se elimina demasiado de prisa por conductividad de la chapa del horno, no se dispondrá del calor requerido para que se inicie la reacción. Pero si se dispone de suficiente calor en el revestimiento, la reacción se realizará satisfactoriamente. Cuando sucede esto, la temperatura del sinterizado puede aumentar de una manera considerable por encima de la que tiene la superficie del revestimiento.

Creo conveniente hacer unas aclaraciones. Es evidente que la temperatura medida en un pirómetro metido en el fuego en un determinado punto del revestimiento o de la costra, no mide la temperatura del material que se está cociendo, pudiendo éste estar mucho más frío o mucho más caliente. Y así no es sólo la temperatura alcanzada por una película sobre la superficie del sinterizado, sino la cantidad total de calor disponible, la que determina el grado al cual los componentes del sinterizado se transformarán en compuestos del cemento. Una adecuada transformación se alcanza, normalmente, sólo como resultado de una intensa y completa reacción exotérmica. Esta reacción puede iniciarse en la superficie del clínker a temperatura de incandescencia, pero se extinguirá, a menos que no se disponga en el revestimiento o en la costra de suficiente cantidad de calor.

Supongamos el caso de tener dos revestimientos en uno de los cuales (A) el calor se trans. mite tápidamente a la chapa del horno, y en el otro (B) no se transmite el calor. Ambos pueden mantenerse a una temperatura dada. Ahora bien, para hacer esto, con (A) se necesitará más calor que con (B), para suplir las pérdidas de calor transmitidas por la chapa del horno. En el caso de (B) se retendrá más calor, porque es menor el gradiente de temperatura entre los sucesivos estratos del revestimientos. Es este almacenamiento de calor, el que es importante en las operaciones industriales para provocar y completar la reacción exotérmica.

Por tanto, el revestimiento de la zona de cocción tiene dos finalidades. Para aislar la chapa del horno, naturalmente, pero también, cosa menos conocida, como medio cambiador de calor entre el combustible y el clínker. La capacidad de almacenar calor es esencial para conseguir el comienzo de la reacción exotérmica requerida para producir un clínker de calidad. 